\title{
Weihnachten zum Einheitstarif
}

Stellen Sie sich doch einmal vor, es gäbe ein neues riesiges Kaufhaus mit allen erdenklichen Geschenken für Weihnachten. Sie könnten da alles finden, was Sie sich vorstellen können. Vom kleinsten Alltagsgegenstand bis hin zum teuersten und exklusivsten Luxusartikel. Von allem, soviel Sie nur wollen - aber das Beste kommt erst noch: Denn Sie können alles haben, was Sie wollen, egal wieviel es kostet, egal wie oft. Selbst wenn Sie es schon hätten, Sie dürfen noch mehr kriegen - als einziges müssen Sie beim Betreten dieses gigantischen Kaufhauses einen Eintritt bezahlen. Da sich dieser Konsumpalast in der Mitte der Schweiz befindet, sollen ja alle den gleichen Zugang erhalten, wobei die Eigentümlichkeit jeder einzelnen Region aber berücksichtigt werden sollte. Kein Problem! Man variiert ein bisschen den Eintrittspreis, und schon sind alle zufrieden. Denn wenn man einmal drin steht, dann kann man sich nach Herzenslust umschauen und zugreifen. Es kostet ja quasi nichts mehr, abgesehen von einem kleinen Unkostenbeitrag, der aber angesichts des Angebotes ja kaum mehr ins Gewicht fällt. Also die Devise lautet umschauen und zugreifen. Da man als Neukonsument in diesem Paradies nicht alles wissen kann, was da möglich wäre, gibt es zum Glück überall riesige Hinweis- und Werbetafeln, damit man ja nicht ein Bedürfnis verpassen könnte, vom dem man bis anhin noch gar nicht wusste, dass man es haben könnte! Dies ist aber noch nicht alles. An jeder Ecke werden einem Zeitungen und Zeitschriften angeboten, in denen jedermann nachlesen und bildhaft erkennen kann, welches Konsumgut er noch nicht kennt. Falls sich einer bis dahin noch mit Zurückhaltung verhielt, werden ihm aufwendig inszenierte Fernsehsendungen schon noch aufzeigen können, dass irgendwo in dem gigantischen Angebotsbereich noch etwas zu finden ist, das auch ihn zum Zugreifen verführen kann. Wer da noch widersteht, ist wirklich selber schuld.

A propos «selber schuld»: Würden Sie bei freier Wahl das Kostbare stehenlassen und das Günstigere nehmen? Das ist ja, wie wenn Sie zum Einheitstarif die Ferien buchen könnten und dann, am Ziel angelangt, anstatt sich mit dem Mercedes zur Luxussuite im Fünfsternehotel fahren zu lassen, lieber selber mit dem Fahrrad in die abgelegene Jugendherberge trampeln würden. Wer will das schon, wenn er «seinen Betrag» ja auch einbezahlt hat, dann hat er doch auch das Recht...

Zurück zum Einkaufspalast. Dieser würde eine Weile recht gut funktionieren. Alle wären zufrieden, insbesondere die Zulieferer. Denn sie haben da ja einen fast unerschöpflichen Absatzmarkt. Und falls eine Bilanzkurve nicht mehr eine genügende Wachstumsrate aufzeigt, muss ein weiteres neues Bedürfnis generiert werden, das hilft garantiert, denn die Lust auf mehr kennt ja keine Grenzen.

Trotz all der guten und besten Absichten aller Beteiligten, alle zufriedenzustellen, muss die Leitung des Kaufhauses leider erkennen, dass die Rechnung am Schluss ein Defizit aufzeigt. Die Manager sind da gefordert, eine Lösung zu finden. Jahrelang recherchieren sie, halten Sitzungen und Konferenzen ab und finden dann zu guter Letzt auch den wahren Grund: Die Anbieter im Kaufhaus sind die Schuldigen. Würden nicht so viele so vielen und so viel herausgeben, dann wäre alles anders, und die Rechnung würde prompt aufgehen. Damit die fehlbaren Angestellten das auch wirklich begreifen, dass sie die Schuldigen sind, kürzt man ihnen den Lohn. Aber auch dies soll ja gerecht verlaufen. Allen Beteiligten wird gekürzt, unabhängig davon, ob sie Luxusartikel oder Selbstgebackenes herausgeben, denn es muss ja kollegial abgestützt sein.

So, nun ist alles wieder in Ordnung. Alle können ihre Weihnachtseinkäufe wieder friedvoll aussuchen und, nach Lust und Laune konsumierend, nach Hause schleppen, was ein jedes Herz begehrt. - Welch schönes und leuchtendes Weihnachtsfest!

Frohe Weihnachten für (fast) alle!!!

A. Gosztonyi, Dietikon 


\section{Un Noël au tarif unifié}

Imaginez un nouveau grand magasin avec tous les cadeaux de fin d'année qui puissent être. Vous y trouverez tout ce qui vous plaît, du plus petit objet domestique au plus coûteux et au plus original des articles de luxe. Et cela en nombre inégalé. Le meilleur reste à venir: vous pouvez tout avoir, quel qu'en soit le prix et tant que vous le souhaitez. Vous pouvez même recevoir plus que vous n'avez déjà. La seule chose que vous devez faire, c'est payer une entrée. Ce palais de la consommation se situant au centre de la Suisse, tous y ont en principe accès, bien que les caractéristiques régionales soient prises en compte. Pas de problème! On varie quelque peu le prix d'entrée et tout le monde est content. Car une fois à l'intérieur, on peut regarder et toucher à loisir. Ça ne coûte plus grand chose, mis à part une contribution aux frais administratifs qui, au regard de l'offre présentée, est modeste. La devise est donc: touchez et servez-vous! Etant donné qu'en tant que nouveau consommateur, on ne saurait tout savoir (ça arrive!) dans ce paradis de la consommation, de grandes indications et affiches publicitaires préviennent le chaland de ne pas manquer de combler ses besoins jusqu'au plus petit et au plus insoupçonné. En effet, comment savoir, sinon, ce que l'on pourrait encore avoir? Ce n'est pas tout. Journaux et revues sont à disposition et chacun peut se faire une idée visuelle du bien de consommation qu'il ne connaît pas encore. Le spectateur jusque là un peu réticent est amené à voir des émissions télévisées consciencieusement mises en scène à grands frais pour lui faire trouver, dans ce choix gigantesque, le créneau qui pourrait le tenter. Qui résiste encore ne peut vraiment qu'avoir tort.

A propos d'avoir tort, je vous le demande: $s i$ vous aviez le choix, pencheriez-vous pour le meilleur marché ou le plus cher? C'est comme si vous réservez des vacances à prix unitaire et qu'arrivé à destination, au lieu de la Mercedes vous conduisant vers la luxueuse suite d'un hôtel cinq étoiles, vous choisissiez la bicyclette qui vous mène péniblement à l'auberge de jeunesse à quelques lieues de là. Qui donc serait d'accord, s'il a lui aussi payé «sa part», de ne pas jouir de son droit...

Revenons à nos moutons et à notre palais consumériste. Certes, tout peut marcher sans problèmes pendant un certain temps, au contentement de tous, surtout à celui des sous-traitants, leurs débouchés étant quasiment inépuisables. Et si la courbe du bilan ne répond plus suffisamment au taux de croissance, il suffit de générer un nouveau besoin qui garantit la pérennité des plaisirs.

Malgré les meilleures volontés de tous les participants, la direction du magasin en question doit malheureusement reconnaître que les comptes accusent finalement un déficit. Les gestionnaires sont priés de trouver une solution. Ils ont cherché des années, ont tenu moult séances et conférences pour aboutir enfin à la cause première: les vendeurs sont les coupables. Tout changerait et les comptes seraient vite équilibrés s'ils ne vendaient pas autant, à autant de personnes. Afin qu'ils comprennent bien que ce sont eux les coupables, on coupe dans les salaires. Mais là aussi, sans distinction entre ceux qui offrent des produits de luxe ou ceux qui se contentent de produits maison, la confraternité étant une règle d'or.

Tout est à nouveau dans l'ordre des choses. On peut recommencer à choisir ses cadeaux de fin d'année dans la joie et la bonne humeur. Joyeux Noël à tous (ou à presque tous)!

A. Gosztonyi, Dietikon 